\title{
PREDICCIÓN ESPECTRAL EN REDES INALÁMBRICAS DE RADIO COGNITIVA
}

\section{SPECTRAL PREDICTION IN COGNITIVE RADIO WIRE- LESS NETWORKS}

\section{RESUMEN}

Uno de los grandes desafíos de la radio cognitiva es establecer la forma adecuada para realizar predicciones en las diferentes fases que componen un radio cognitivo. A partir de las estimaciones se pueden determinar el estado y características de los canales, la actividad de los usuarios primarios y secundarios, la movilidad espectral; todo ello con el fin de que los nodos no licenciados puedan aprovechar adecuadamente y de manera oportunista las bandas subutilizadas. En este artículo se presenta una revisión de algunas de las técnicas más relevantes que han sido aplicadas en la predicción espectral en la radio cognitiva.

Palabras clave: radio cognitiva, algoritmos, red neuronal, lógica difusa, regresión lineal, etapas de la radio cognitiva.

\begin{abstract}
One of the great challenges of cognitive radio is to establish the correct way to make future predictions in the different phases in a cognitive radio. From estimates can determine the status and characteristics of the channels, the activity of the primary and secondary users, the spectral mobility; all this in order that the nodes are not properly licensed and can take advantage of underutilized bands opportunistic way. This article reviews some of the most relevant techniques that have been used in spectral prediction in Cognitive Radio is presented.
\end{abstract}

Keywords: Cognitive radio, algorithm, neural network, fuzzy logic, linear regression, cognitive radio stages.
Danilo Alfonso López Sarmiento

Magister en Ciencias de la Información y las Comunicaciones

Docente planta de la Universidad Distrital Francisco José de Caldas dalopezs@udistrital.edu.co Bogotá, Colombia

\section{Edwin Rivas Trujillo}

Doctor en Ingeniería Eléctrica, Electrónica y Automática

Docente planta de la Universidad Distrital Francisco José de Caldas erivas@udistrital.edu.co Bogotá, Colombia

\section{Oscar Gualdrón Guerrero}

Descripción.

oscar.gualdron@unipamplona.edu.co Pamplona, Colombia

Tipo: Revisión de tema

Fecha de Recepción: Junio 25 de 2014

Fecha de Aceptación: Octubre 25 de 2014 


\section{INTRODUCCIÓN}

La tecnología de radio cognitiva (CR) posibilita que los usuarios secundarios (SU) compartan el espectro licenciado con los nodos primarios (PU) sobre la base de la no interferencia y soportado en las funciones que componen la CR (apartado 2). De acuerdo con [1] [2], se presentan falencias que dificultan la capacidad de mejoramiento de las redes, entre las que se destacan:

- En la detección; el sensado en espectros de banda ancha puede resultar en retardos de tiempo no despreciables [1] [2], sobre todo en los sitios donde existe una alta actividad.

- En la toma de decisiones; donde sólo selecciona y asigna bandas después de que sean solicitadas por el SU, variable que quebranta la eficiencia de la red en razón a los retrasos introducidos.

- En la compartición espectral; los usuarios cognitivos pueden arribar a la red con diferentes requerimientos de calidad de servicio (QoS) y ancho de banda. Asignar espectro a solicitudes heterogéneas puede conducir también a considerables retrasos en el tiempo [1].

Las falencias citadas dependen directamente de los modelos o metodologías que se utilicen para realizar estimaciones futuras de comportamiento y repercuten de manera negativa en el óptimo funcionamiento de las redes. Por tal motivo es necesario que los paradigmas de predicción espectral sean inteligentes, autónomos, ya que redundaría en una disminución significativa del consumo de energía al requerir menor procesamiento computacional. El artículo presenta cómo en la literatura científica se aplican diferentes paradigmas de programación en la selección de espectro, partiendo de la definición formal de radio cognitiva, posteriormente se presentan algunas técnicas utilizadas en la predicción, y se finaliza las respectivas conclusiones.

\section{RADIO COGNITIVA (CR)}

La CR es considerada la tecnología que podría mejorar la administración del espacio radio- eléctrico útil en las transmisiones inalámbricas de datos, fundamentada en la asignación de frecuencias de forma dinámica, dependiendo de la actividad de las bandas espectrales, y logrando que usuarios licenciados (PU) y no licenciados (SU) coexistan en la misma infraestructura de red. El marco de gestión espectral en CR plantea cuatro funciones: sensado, decisión, movilidad y compartición de espectro (figura 1).

La detección consiste en la identificación de las oportunidades espectrales y la capacidad de detectar en tiempo real la aparición de usuarios autorizados en el agujero espectral. La toma de decisiones se refiere a la capacidad de los nodos no licenciados para seleccionar la mejor banda disponible en términos de la calidad de servicio solicitada por los SU y de acuerdo con las características existentes en la banda. Por su parte, la movilidad espectral es la habilidad de un nodo CR para desocupar el medio de transporte cuando se detecta la presencia de un PU desde la fase del sensado, y a su vez la capacidad de desplazarse a otra banda libre haciendo un handoff reactivo o proactivo; la compartición de espectro controla el acceso coordinado al canal seleccionado por los SU [1].

Con base en la figura 1, el funcionamiento del ciclo cognitivo en un nodo (que es el que integra las diferentes etapas de la radio cognitiva) parte de un barrido de frecuencia en el que funciona el dispositivo, buscando bandas en desuso mediante monitorización; información que es entregada a la etapa de decisión para que puedan ser seleccionados y asignados a los SU. La distribución de bandas depende de la topología de la red; si es centralizada es realizada por el dispositivo de acceso (estación base o un dispositivo central); si es ad hoc, de manera distribuida los SU se comunican entre sí sin ningún tipo de intermediario, tomando una decisión de forma cooperativa. Debido a la dinámica propia de la $\mathrm{CR}$, existe la posibilidad de que más de un SU desee acceder al espectro, segmentación que es resuelta por la compartición espectral; si durante la transmisión no se alcanza a completar la transferencia porque de forma probabilística arriba un PU a utilizar la banda que se cedió o si hay una disminución en las prestaciones a nivel de la QoS (en razón 


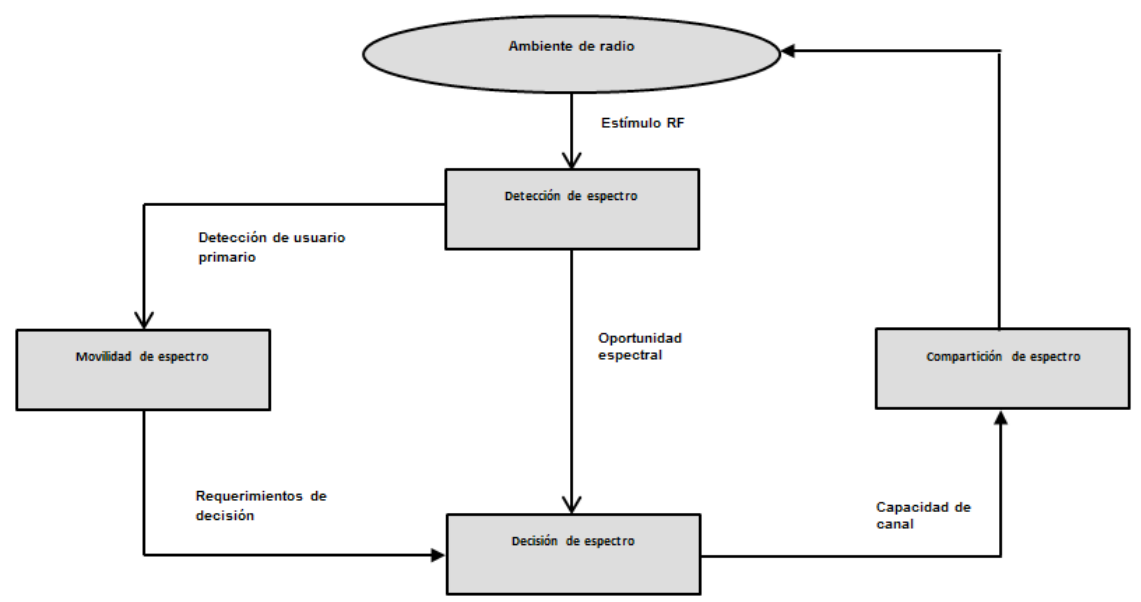

Figura 1. Marco de la gestión espectral en CR [1]

a que el SU no está pagando por ese recurso), debe abandonar el canal y movilizarse a otro. En este sentido hay una probabilidad de que se tenga que realizar una movilidad espectral, siendo necesario volver a realizar un sensado, para determinar a dónde debe darse el movimiento. Teniendo en cuenta que cada una de las etapas que componen el ciclo cognitivo en CR aumenta los tiempos de procesamiento por las complejas tareas que son necesarias desarrollar y predecir (sensado, estimación futura, división espectral, comportamiento aleatorio de SU y PU, entre otros), desde sus inicios la CR ha venido trabajando con el concepto de predicción de espectro [1], problema desafiante que implica varios subtemas, tales como la estimación del estado del canal, de la actividad de los PU, del comportamiento de llegada de los SU a la red, de la selección y asignación previa de los canales, de las características para determinar caminos de respaldo en caso de la necesidad de hacer handoff espectral.

\section{TÉCNICAS UTILIZADAS EN LA RADIO COGNITIVA}

\subsection{Predicción basada en la ocupación histórica del canal}

En [3] se propone un algoritmo de decisión de espectro basado en predicciones a partir del análisis y registro de la ocupación histórica del canal, que incluye la opción de establecer la probabilidad de llegada de un cliente licenciado antes de que el SU termine con la sesión de envío generada desde los nodos cognitivos.
La aplicación utiliza un método de aprendizaje en línea que actualiza periódicamente los parámetros del sistema. El transmisor/receptor puede detectar el espectro libre en un amplio rango, pero tiene la capacidad de seleccionar solamente uno. El modelo de predicción asume el comportamiento ON/OFF similar a [4] y utiliza una estructura en árbol invertido (figura 2) para almacenar y mantener la información histórica del canal, pero establecida para que funcione de acuerdo a la frecuencia de aparición y no a la probabilidad de aparición (ecuación (1)).

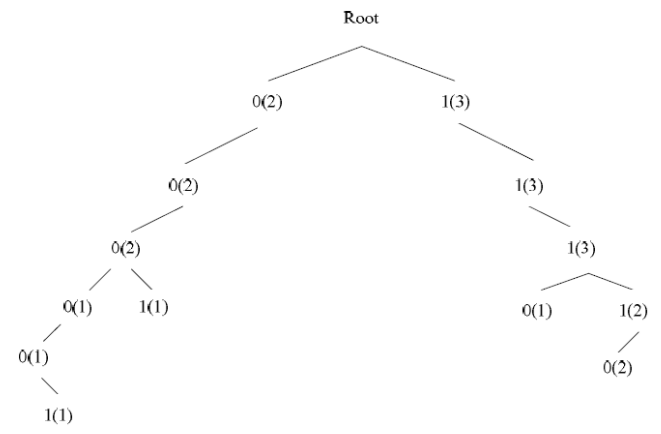

Figura 2. Estructura en árbol que registra y mantiene la actividad histórica registrada [3]

$P_{n}=p\left(S_{i}(K+n)\right)=0 \mid S_{i}(K)$
$P_{n}=0, \ldots, S_{i}(K+n-1)=0=\frac{f_{n+1}(0)}{f_{n+1}(0)+f_{n+1}(1)}$

Donde $\operatorname{Si}(k)$ representa la serie binaria; $f_{n+1}(0)$ es la frecuencia de aparición en la rama izquierda del árbol en la capa $(n+1) ; f_{n+1}(1)$ es la frecuencia en la rama derecha del árbol en la misma capa. 


\subsection{Predicción basada en la carga de la batería de la red}

En [5] se expone una predicción espectral "consciente" cuando el nivel de batería de la red cae por debajo del umbral de funcionamiento normal, incluyendo una QoS aceptable (para requisiciones de ancho de banda) y prioritaria para servicios de voz, video, a través de medios que requieran un consumo de potencia baja de transmisión. La propuesta selecciona primero un canal que satisfaga las necesidades de los datos con prioridad más alta; cumplida esa tarea, entra en funcionamiento el control de admisión a administrar el acceso de los paque- tes de más baja prioridad que vayan a competir por el ancho de banda disponible. La interconexión de los bloques que hacen parte activa del diseño se muestra en la figura 3.

El CSMU basado en la información entregada por la unidad de control de QoS (QCU) y soportado en la unidad de monitoreo energético (EMC) (que detecta si el radio está funcionando con alimentación AC o con batería), estima y predice el canal con las condiciones establecidas desde la capa de aplicación. El canal de radio frecuencia y el de monitoreo evalúan constantemente e informan acerca de los cambios que se van generando en el medio ambiente.

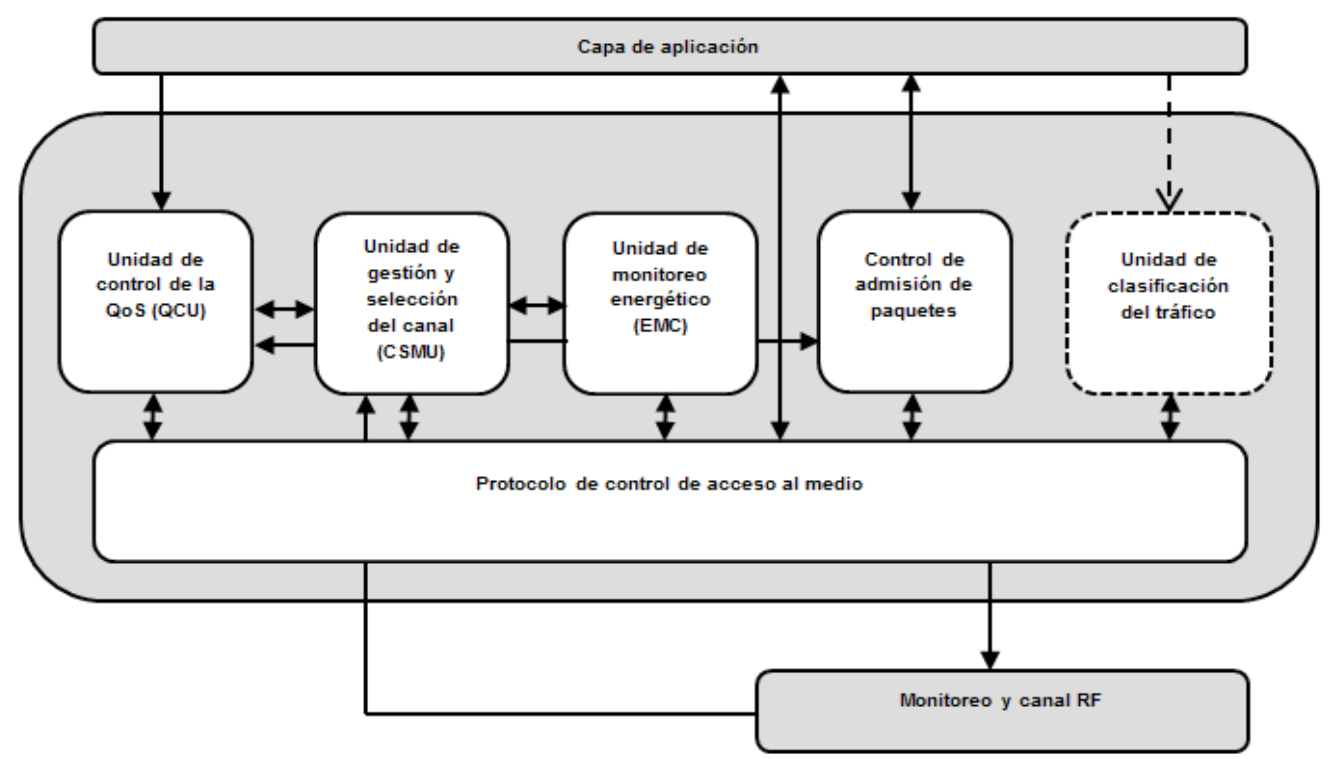

Figura 3. Diagrama de bloques de estimación bajo condición de estado crítico de disponibilidad de energía en las baterías [5]

\subsection{Predicción basada en datos medidos}

En [6] se propone una técnica para predecir el espectro con estadísticas espaciales y campos aleatorios usando datos obtenidos de medidas espectrales (figura 4). Se caracteriza el espectro estudiando su comportamiento como función de un campo aleatorio desconocido, que no son más que métodos considerados como extensiones de la teoría de procesos estocásticos desde un espacio unidimensional a uno multidimensional. Se utilizan las estadísticas de Moran [7] y Geary [8] para tomar las medidas tendientes a caracterizar la estructura espacial estocástica de segundo orden del espectro licenciado para su estimación futura, midiendo el grado de correlación para datos espaciales cuya estructura de covarianza es definida por vecindades [9].

Los modelos estadísticos de Moran y Geary fueron probados en medidas de ocupación distribuida de espectro de bandas de frecuencia para servicios inalámbricos de $\mathrm{Wi}-\mathrm{Fi}$, TV y redes celulares. No obstante, se concluyó que Geary requiere una cantidad de datos suficientes para asegurar que patrones similares no sean descartados cuando las bandas son raramente monitorizadas. 


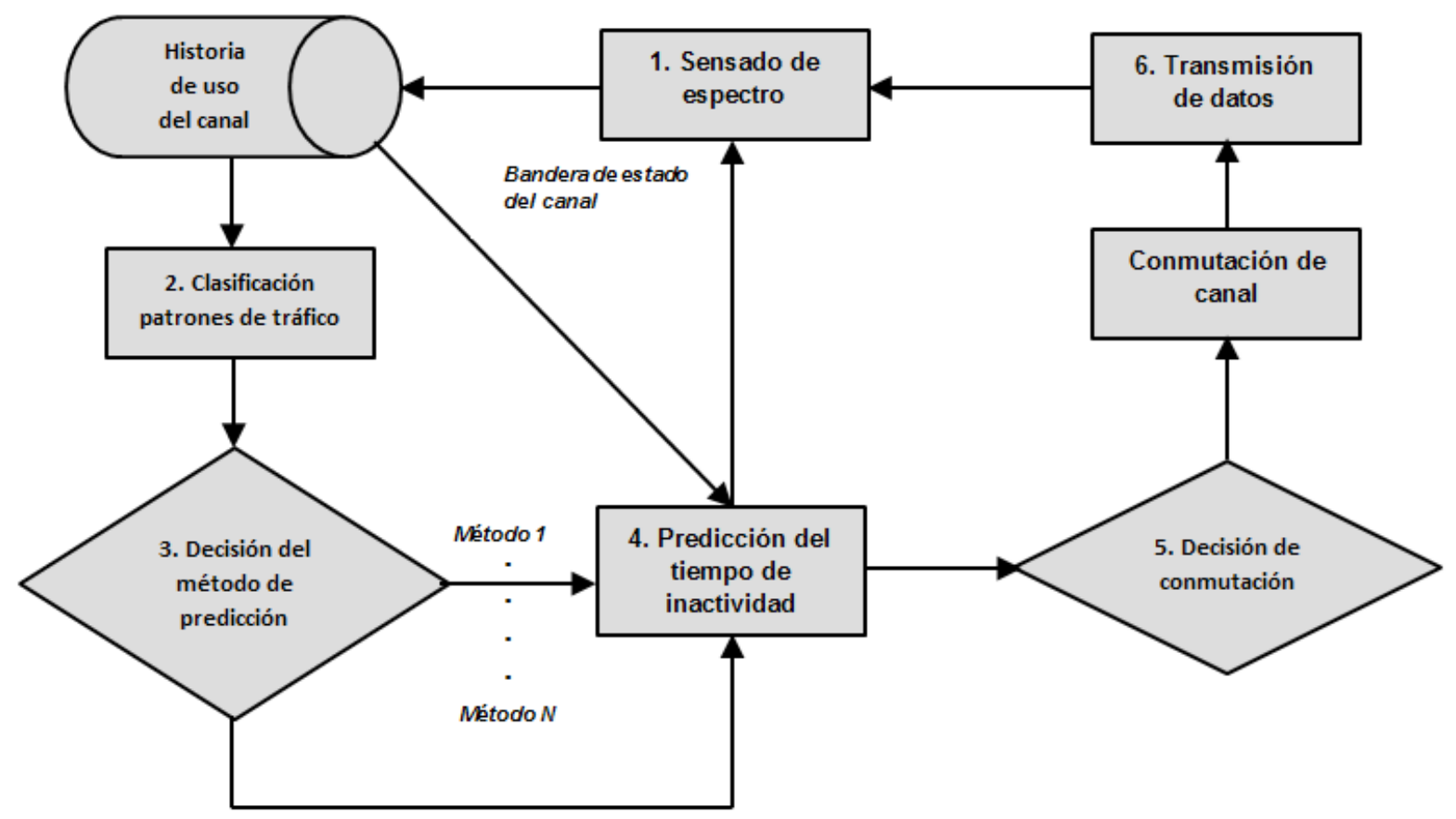

Figura 4. Modelo del sistema de predicción [9] [10]

\subsection{Esquema basado en predicción de correlación}

Un modelo de predicción del espectro es propuesto en [11] y pronostica el estado futuro del canal usando el histórico de detección obtenido de los resultados de decisión locales. El propósito de la correlación fundamentada en esquemas de predicción beneficia la correlación a lo largo del estado histórico de la ventana de espectro. En primera instancia, el coeficiente de correlación de Pearson [12] se mide entre las muestras históricas. Luego, si este coeficiente está por encima de un umbral, la predicción de la ventana se llena con la última muestra del histórico de la ventana. Si no se encuentra una correlación suficiente, el procedimiento llena la ventana con el resultado común. El pronóstico intuitivo sobre el estado futuro del espectro se calcula si existe una alta correlación entre las muestras del histórico; para las próximas ranuras el comportamiento del espectro muy probablemente será igual al último estado.
Este procedimiento se plantea en el diagrama de flujo de la figura 5, con base en el algoritmo presentado en [11], donde es el histórico de la ventana $\left(W_{H}=\left[H_{1}, H_{2}, \ldots, H_{/ W_{H}}\right]\right), x$ el índice del vector $\left(1,2, \ldots, / W_{H} /\right)$, $\delta c$ el umbral de correlación, el tamaño de la ventana. Como se puede deducir del dia grama de flujo, el programa visualiza la capacidad de estimación o no del estado futuro de un rango de frecuencias. Para ello obtiene los valores de $W_{H}$ y $\delta c$, a partir de allí se carga el valor de los elementos históricos $W_{H}$, luego se divide cada elemento del vector $W_{H}$ entre el valor más grande de un método conocido como de las mayorías. Si el resultado de esta división es mayor al umbral de correlación que se da, el algoritmo es capaz de predecir el estado futuro del canal llenando los demás elementos del vector $W_{H}$ con ese resultado, asignándolo al SU que lo requiera; si no se supera ese umbral, no es posible predecirlo y se llenan los demás elementos del vector $W_{H}$ con el resultado promedio de las divisiones. 


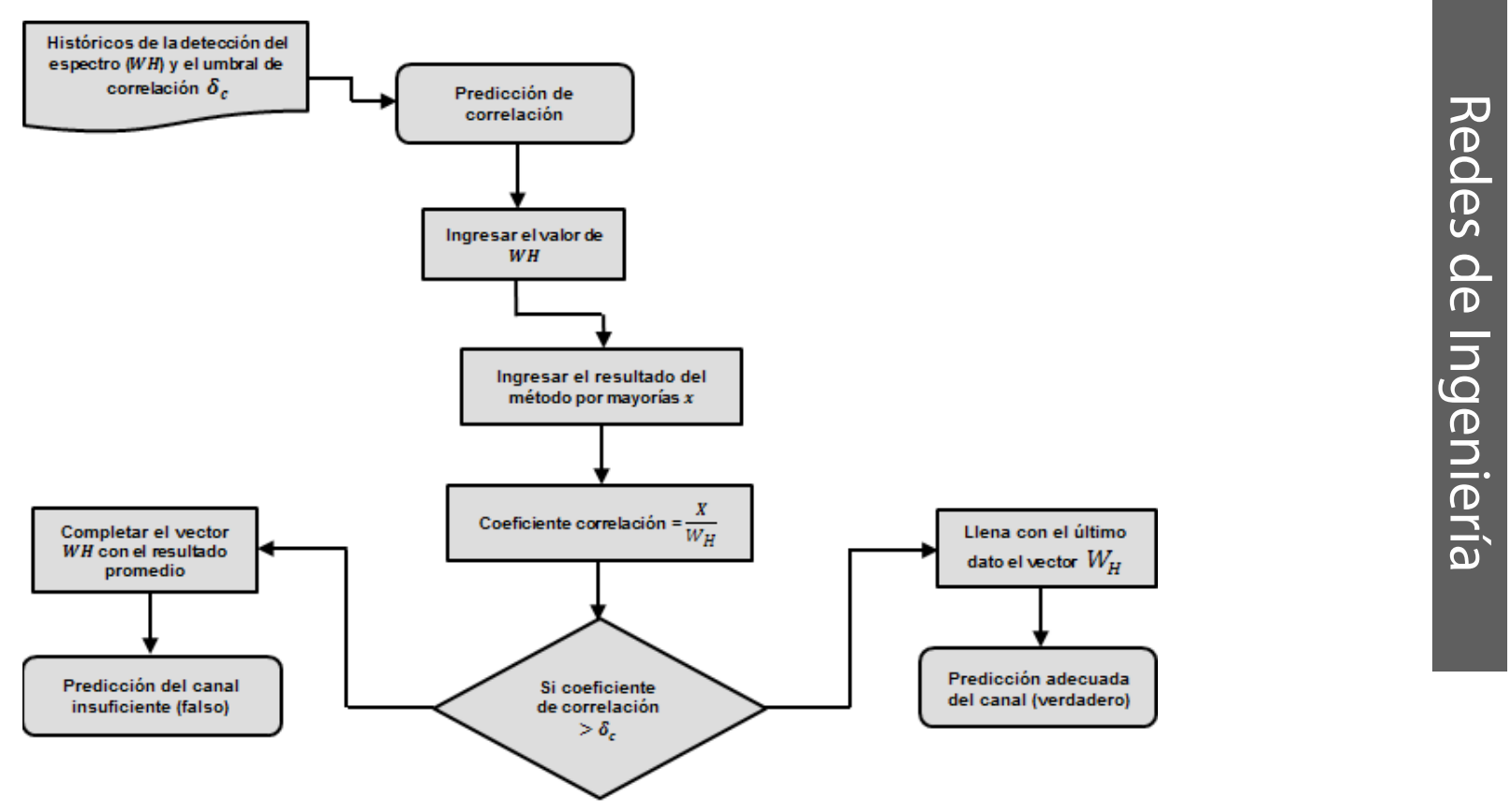

Figura 5. Diagrama de flujo con base en el método de predicción de correlación (elaboración de los autores a partir del algoritmo [11])

\subsection{Método de predicción basado en regresión lineal}

En [11] se establece que el coeficiente de correlación de Pearson es un índice estadístico que mide la relación lineal entre dos variables cuantitativas y su cálculo se obtiene relacionando la covarianza con el producto de las desviaciones estándar. Por lo tanto, el coeficiente de Pearson da una idea acerca de la relación lineal entre el estado del sensado espectral y el vector índice (dado por $X=\left[1,2, \ldots, / W_{H} /\right]$ ); donde es la posición de memoria que almacena el histórico de las decisiones espectrales). Si esta relación supera un umbral, puede ser usada en la predicción espectral, para obtener la estimación. La figura 6 presenta el proceso seguido por [11] para realizar la predicción del canal fundamentado en la correlación y regresión lineal, donde se observa que el funcionamiento del algoritmo inicia por establecer el valor del vector histórico de la ventana $W_{H}$ y por conocer el valor del umbral $\delta c$.

Su funcionamiento inicia por cargar el valor de los elementos históricos de la ventana $W_{H}$, después relaciona cada elemento del vector entre el valor más grande del método de las mayorías; si el resultado de esta división es mayor al umbral (que es variable y en este instante igual a 0,5$)$, el vector $W_{H}$ se llena con binarios y se dice que el algoritmo es capaz de predecir el estado futuro del canal, de lo contrario no se puede predecir el estado y se llena $W_{H}$ con el elemento mayor de las divisiones. 


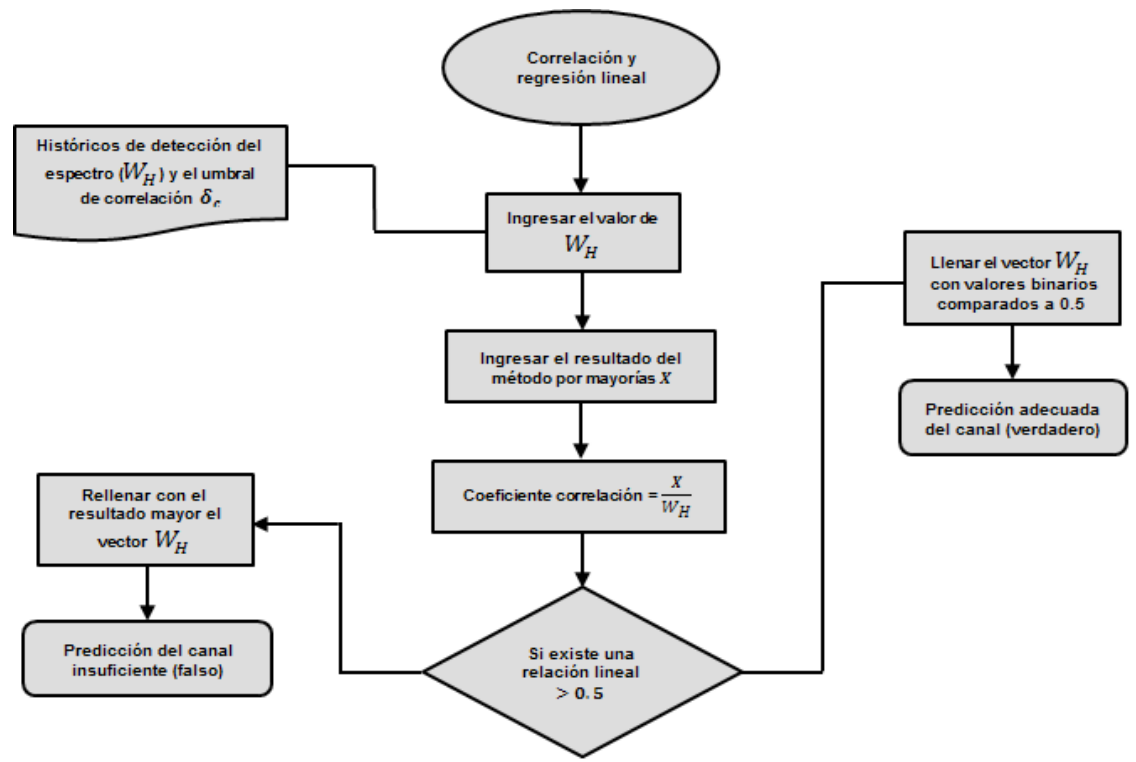

Figura 6. Diagrama de flujo usando regresión lineal (elaboración de los autores a partir del algoritmo [11])

\subsection{Método predictivo con lógica difusa}

En [13] se utiliza la lógica fuzzy para resolver los problemas en el manejo del espectro relacionados con la ubicación de canales específicos y decisión de uso, dentro del espectro hacia el usuario secundario. El sistema difuso jerárquico consiste en dos controladores difusos [14] [15], planteados como aparece en [16] y [11] e implementados de acuerdo con lo mostrado en el diagrama de flujo de la figura 7. El FLS1 (Fuzzy Logic Systems)recibe tres entradas, la eficiencia espectral, distancia y movilidad, y entrega al FLS2 la probabilidad de utilización del canal. FLS2 funciona dependiendo de la información de la utilización del canal, la tasa de habilitación del canal, y la tasa de no disponibilidad del canal, y define la posibilidad de asignar un canal para el acceso del usuario secundario al espectro. En el FLS1 se estima la utilización del canal primario y se decide si éste puede ser usado por un secundario, resultado que es entregado al FLS2 junto con las variables previamente mencionadas para estimar la probabilidad de asignar el canal. Después de la selección del canal, la tasa de error de bit (estimado) (BER) se obtiene de la ecuación (2).

$$
B E R_{\text {estimado }}=\frac{B E R_{S} \cdot C_{S}+B E R_{P} \cdot C_{P} \cdot U_{\text {estimada }}}{C_{S}+C_{P}}
$$

Donde el $B E R_{S}$, es la tasa de error de bit del canal secundario, $B E R_{P}$ la tasa de error de bit del canal primario, $C_{S}$ la capacidad máxima del canal para el canal secundario, y $C_{P}$ la capacidad máxima del canal para el canal primario y la $U_{\text {estimada }}$ está dada por la ecuación (3).

$$
U_{\text {estimada }}=\frac{T_{\text {on }}}{T_{\text {off }}}
$$

Donde $T_{o n}$ es el periodo durante el cual el canal no está llevando tráfico (disponible) y $T_{\text {off }}$ es el período durante el cual el canal está llevando tráfico (no disponible). El ciclo final del algoritmo establece si se puede cumplir con la QoS solicitada por el SU. 


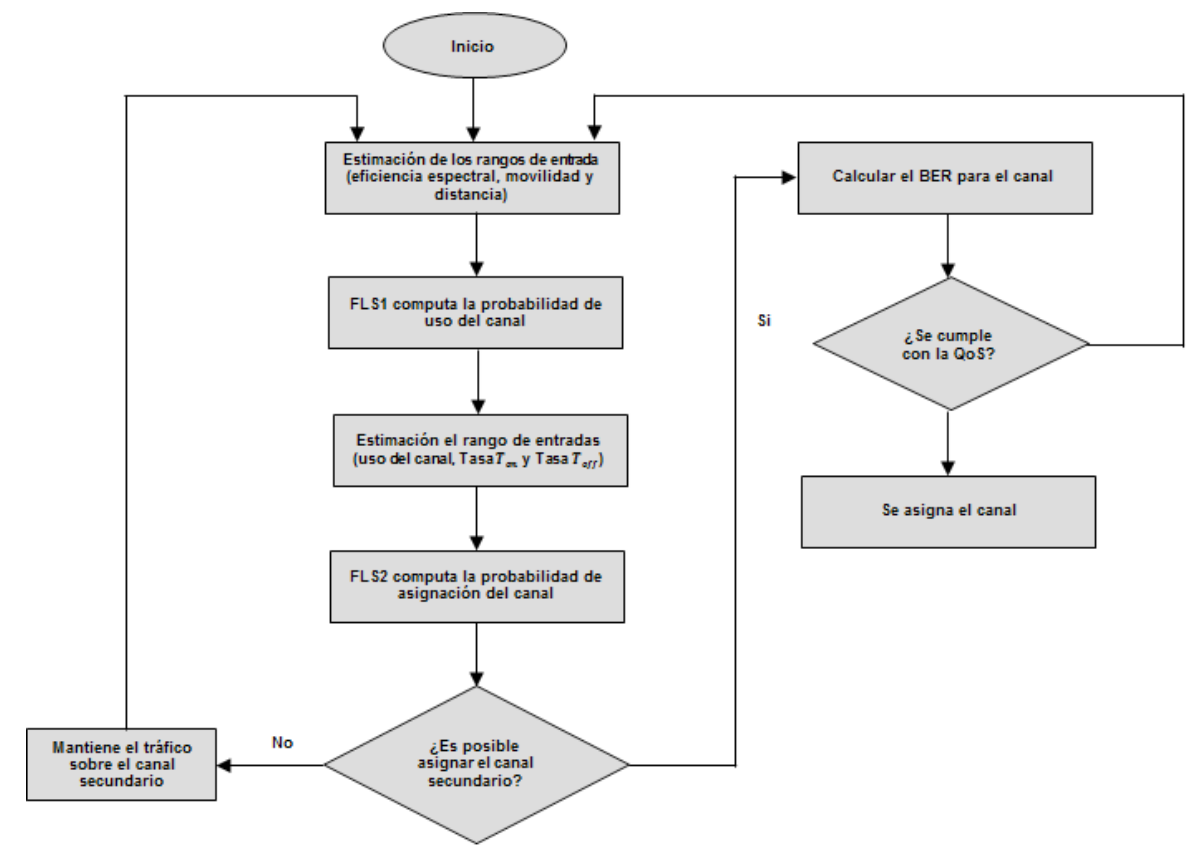

Figura 7. Diagrama de flujo para la estrategia de predicción y asignación de canal basada en lógica difusa [13]

\subsection{Método de predicción con perceptron multicapa}

Una red MLP es una estructura multicapa no lineal consistente de una o más entradas, una salida y varias capas ocultas (figura 8). Excluyendo la capa de entrada, cada nodo restante contiene un cierto número de unidades computacionales (neuronas) que calculan la suma ponderada de la entrada y transforma ésta a través de una función de activación no lineal $\Gamma$. De acuerdo con la figura 8, las variables de orden son $n_{i}^{l-1}$ (en la que l-1 hace referencia a la capa anterior e $i$ corresponde al número del nodo de esa capa), $n_{j}^{l}$ (donde l es la capa entrante y $j$ el número del nodo de esa capa), $W_{i j}^{l-1}$ (en la que $W$ es el peso de la salida entre el nodo $i$ y el nodo $j$ ), $y_{i}^{l-1}$ (que es la variable que hace referencia a la salida del nodo anterior), $y_{j}^{l}$ (es la salida del nodo de la capa oculta, y es quien da la suma ponderada de la entrada $\Gamma)[1]$.

A partir de la definición conceptual de multicapa, en [18] se diseña un predictor de espectro MLP que no requiere un conocimiento a priori de las características del PU. En su enfoque, cada usuario CR predice los futuros estados del canal mediante el uso de un predictor basado en MLP y detecta solamente aquellos canales que están inactivos (lo que reduce el consumo de energía de los usuarios RC). La transformación no lineal del diseño del predictor fue generada a partir de una función tangente hiperbólica, donde las neuronas pertenecientes a diferentes capas están conectadas a través de pesos adaptativos. La salida de la neurona $j$ en la capa $l^{\text {th }}$ denotada por $y_{j}^{l}$ se representa por la ecuación (4).

$$
y_{j}^{l}=\frac{1-\exp \left(-v_{j}^{l}\right)}{1+\exp \left(-v_{j}^{l}\right)}
$$

Donde $v_{j}^{l}=\sum_{i} y_{j}^{l-1} w_{j i}^{l}$ es la suma de los pesos (de las entradas) provenientes de la salida de las neuronas en la capa $(l-1)^{\text {th }}$ usando los pesos adaptativos $w_{j i}^{l}$ conectando la neurona $j$ en la capa $l^{\text {th }}$ y neurona $i$ en la capa $(l-1)^{\text {th }}$. El número de capas ocultas y de neuronas en cada capa dependen de la aplicación. En este caso en particular, se concluyó [18] que dos capas ocultas eran suficientes para el predictor MLP (la primera de ellas formada por 15 neuronas y la segunda, por 20). La capa de salida solamente estaba integrada por una neurona, lo que establecía el orden del predictor MLP en 4. 


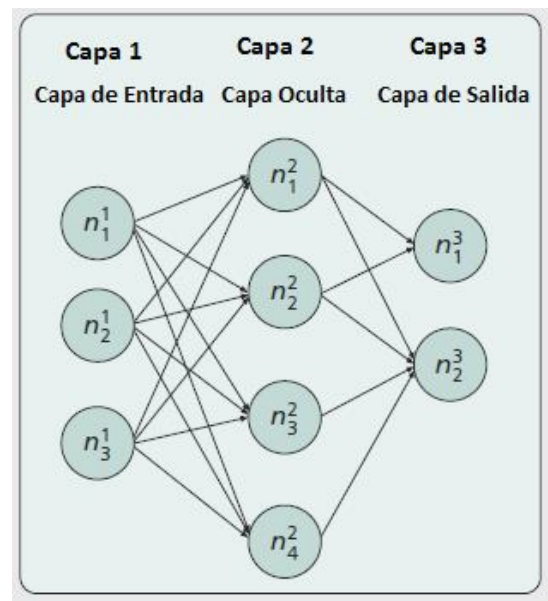

(a)

$n_{i}^{l-1}$ :El nodo i-ésimo en la capa L-1

$n_{j}^{l}$ : El nodo j-ésimo en la capa L

$w_{i j}^{l-1}$ : La conexión entre

el peso del nodo $n_{i}^{l-1}$ y el nodo $n_{j}^{l}$

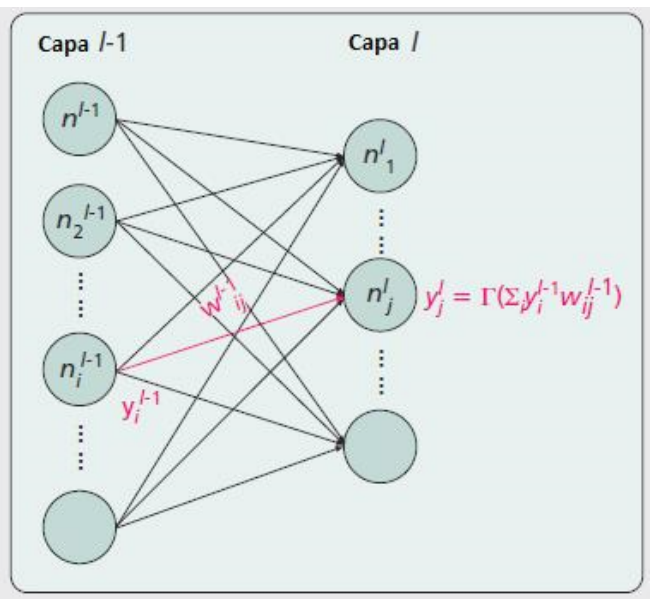

(b) $y_{i}^{l-1}$ : la salida del nodo $n_{i}^{l-1}$

$y_{j}^{\prime}$ : la salida del nodo $n_{j}^{l}$

$\Gamma(\cdot)$ : Una activación de función no-Lineal

Figura 8. Perceptrón multicapa en redes neuronales: a) ejemplo de un modelo de red neuronal, b) proceso de computación de una neurona $n_{j}^{l}[1]$

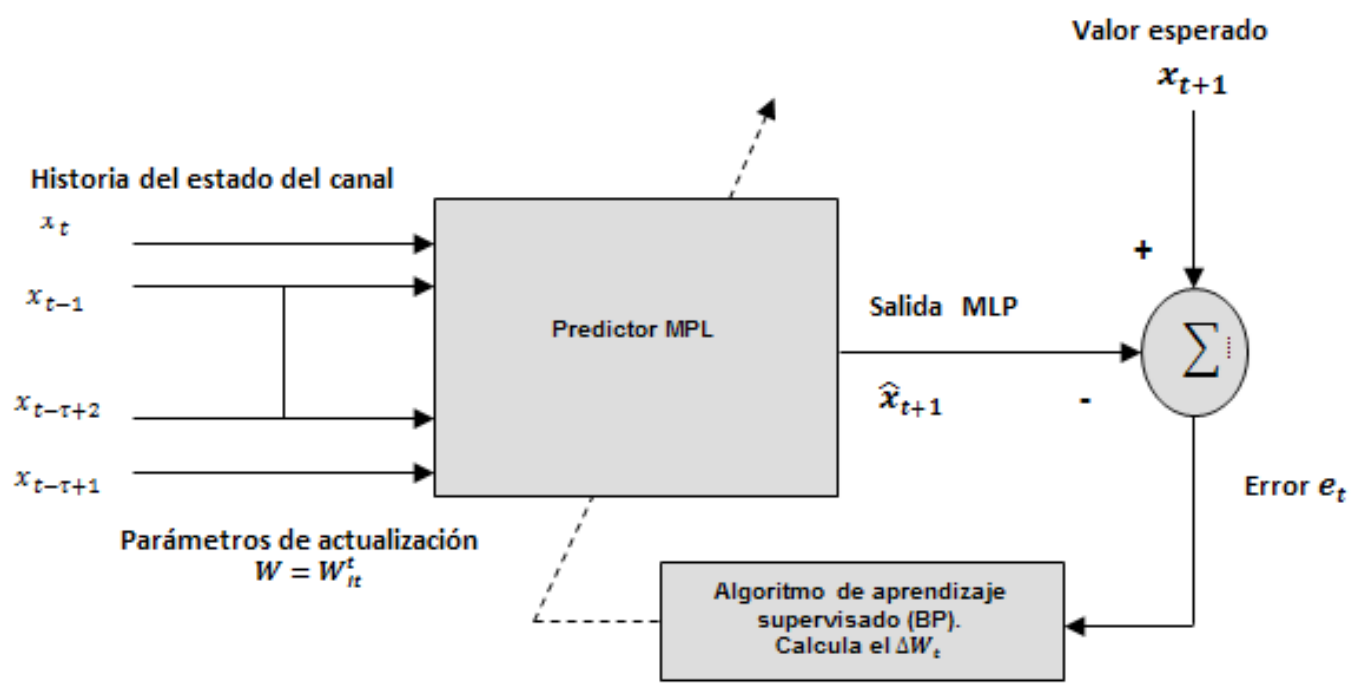

Figura 9. Entrenamiento del predictor MLP [19]

El proceso de entrenamiento de la red se muestra en la figura 9, donde los parámetros del predictor se actualizan utilizando el algortimo de aprendizaje supervisado (BP) [19], para ajustar el nivel de error a la salida del sistema al valor más pequeño. Los patrones de entrenamiento se obtienen ordenando la totalidad de las series binarias $x_{1}^{T}$ dentro del vector de en- trada $x_{t-\tau+1}^{t}=\left\{x_{t}, x_{t-1}, \ldots, x_{t-\tau+1}\right\}$ de longitud $\tau$ y el correspondiente valor deseado $x_{t+1}$.

Para cada vector de entrada a la MLP, el resultado entregado por las neuronas $\left(\hat{x}_{t+1}\right)$ se calcula (desde la primera capa oculta hasta la última) usando la ecuación (4). La diferencia entre el valor deseado $x_{t+1}$ y el estimado $\hat{x}_{t+1}\left(e_{t}\right)$ está 
dada por la ecuación (5).

$$
\begin{aligned}
& e_{t}=x_{t+1}-\hat{x}_{t+1} \\
& e_{t}=x_{t+1}-y_{1}^{0}
\end{aligned}
$$

El entrenamiento del algoritmo tiene como objetivo minimizar el valor $e_{t}$, lo que redundará a su vez en una predicción acertada.

\section{CONCLUSIONES}

El presente artículo revisa las técnicas aplicadas a la estimación espectral, entre las que se destaca el paradigma MLP (en la fase de sensado), el cual es una metodología de entrenamiento y autoaprendizaje que tendría la posibilidad de

\section{Referencias}

[1] X. Xiaoshuang, J. Tao, C. Wei, H. Yan, and C. Xiuzhen. "Spectrum prediction in cognitive radio networks", IEEE Wireless Communications, vol. 20, no. 2, pp. 9096, 2013.

[2] Z. Lin, X. Jiang, L. Huang, and Y. Yao "A energy prediction based spectrum sensing approach for cognitive radio networks", International Conference on Wireless Communications, Networking and Mobile Computing (WiCom), Beijing, September, 2009.

[3] L. Yang, D. Yuning, Z. Hui, Z. Haitao, S. Haixian, and Z. Xingxing, "QoS provisioning spectrum decision algorithm based on predictions in cognitive radio networks", Wireless Communications Networking and Mobile Computing (WiCOM), 6th International Conference on IEEE, p.p. 1-4, Chengdu, Sept. 2010.

[4] T. Samer and W. Li-Chun, "Loadbalancing spectrum decision for cognitive radio networks with unequalwidth channels", Vehicular Technology Conference (VTC Fall), IEEE, pp. 1-5, Quebec City, Sept. 2012.

[5] M. Vishram, C. Lau, Ch. Syin, and K. Ashish, "Energy aware spectrum decision aprender de los errores del pasado, corregirlos $\mathrm{y}$ aplicarlos al tomar decisiones futuras.

La estimación eficiente es una de las temáticas de investigación relevantes en las redes de radio cognitiva porque de ellas depende gran parte del funcionamiento óptimo del sistema. No obstante, a partir de la literatura existente se hace necesario el desarrollo de esquemas de estimación más acertadas, incluyendo nuevas metodologías como aprendizajes de máquina (ML), máquinas de soporte vectorial (SVM) y procesos jerárquicos analíticos con lógica difusa (FAHP), sobre todo en las etapas de decisión y compartición de espectro que aún siguen siendo críticas por la escasez de propuestas.

[6] J. Riihijarvi, P. Mahonene, M. Wellens, and M. Gordziel, "Characterization and modelling of spectrum for dynamic spectrum access with spatial statistics and random fields", IEEE 19th Int. Symposium on Personal, Indoor and Mobile Radio Communications, Cannes, Sep. 2008.

[7] P. Moran, "Notes on continuous stochastic phenomena", Biometrika, vol. 37, no. 1, pp. 17-23, 1950.

[8] R. Geary, "The contiguity ratio and statistical mapping", The Incorporated Statistician, vol. 5, no. 3, pp. 115-145, Nov. 1954.

[9] M. Masonta, M. Mzyece, and N. Ntlatlapa, "Spectrum decision in cognitive radio networks: A survey", IEEE Communications Society, Communications Surveys \& Tutorials, vol. 15, no, 3, pp. 1088-1107, July 2013.

[10] M. Hoyhtya, S. Pollin, and A. Mammela, "Improving the performance of cognitive radios through classification, learning, and predictive channel selection", Advances 
in Electronics and Telecommunications, vol. 4, no. 2, pp. 28-38, Dec. 2011.

[11] G. Uyanik and B. Canberk, "Predictive spectrum decision mechanisms in Cognitive Radio Networks", IEEE Globecom Workshops (GC Wkshps), pp. 943-947, Anaheim, Dec. 3-7, 2012.

[12] B. Canberk, I. Akyildiz, and S. Oktug, "Primary user activity modeling using first-difference filter clustering and correlation in cognitive radio network", IEEE/ACM Transaction Networking, vol. 19, no. 1, pp. 170-183, Feb. 2011.

[13] M. Lindberg, "QoS Provisioning in Channel Allocation Strategy in Cognitive Radio Using Fuzzy Logic", American Journal of Electrical Systems, vol. 2, no. 1, pp. 11-22, 2012.

[14] G. Klir and B. Yuan, Fuzzy sets and fuzzy logic: theory and applications. Upper Saddle River, NJ, USA: Prentice-Hall PTR, 1995.

[15] J. Jantzen (1998, Aug.) Design of Fuzzy
Controllers [En línea], consultado en mayo 25 del 2014. Disponible en http:// faculty.petra.ac.id/resmana/private/fuzzy/ design.pdf

[16] L. Ming-Ling, H. Chung, and Y. FangMing "Modeling of hierarchical fuzzy systems", Elsevier Fuzzy Sets and Systems, vol. 138, no. 2, pp. 343-361, Sept. 2003.

[17] L. XinWang "Analysis and design of hierarchical Fuzzy Systems", IEEE Transactions On Fuzzy Systems, vol. 7, no. 5, pp. 617-624, Oct. 1999.

[18] V. Tumuluru, P. Wang, and D. Niyato, "A Neural Network Based Spectrum Prediction Scheme for Cognitive Radio", IEEE International Conference on Communications (ICC), Cape Town, May 2010.

[19] S. Haykin, Neural Networks: $A$ comprehensive foundation, 2nd ed. Upper Saddle River, NJ USA: Prentice Hall, 1999. 